\title{
Positional and sleep dyspnoea due to posterior exophytic ependymoma of the medulla oblongata
}

A 43 year old man suffered several episodes of dyspnoea occurring during sleep and while awake in the supine position; no respiratory disturbances were reported when standing or sitting. Two weeks later, headache and vomiting also occurred. At observation, 1 month after the clinical onset, the neurological examination revealed bilateral papillo-oedema. There were no abnormalities of the heart, upper respiratory pathways, or lungs. Spirometric and blood gas studies were normal. Brain magnetic resonance imaging (MRI) showed tetraventricular hydrocephalus and a posterior fossa tumour arising from the posterior aspect of the medulla and occupying the cisterna magna (fig 1); the tumour mass had regular margins and intense contrast enhancement. Because of the sudden visual loss, an urgent endoscopic third ventriculostomy was performed, and improvement of the visual function resulted. Six days later the patient underwent microsurgical suboccipital craniectomy in the sitting position. A round, reddish, vascular tumour was found in the cisterna magna arising from the posterior aspect of the medulla, which was infiltrated. The mass was initially reduced; however, the gentle upward retraction of its inferior pole, in order to expose its attachment, resulted in severe desaturation and bradycardia, which promptly resolved. Tumour removal was then accomplished. Postoperatively the patient showed transient diplopia and ataxia, with no episodes of dyspnoea. The histological examination indicated a "clear cell" ependymoma with moderate mitotic index. Brain MRI showed absence of residual tumour in the medulla. Currently, 4 years after surgery, the patient is symptom free, with no tumour recurrence.

Sleep apnoea has been reported in association with various neurological disease in the posterior fossa, ${ }^{1}$ including intraaxial brainstem tumours ${ }^{2-6}$ such as gliomas, ${ }^{35}$ gangliogliomas, ${ }^{46}$ and lipomas. ${ }^{2}$ In all cases there was variable tumour infiltration of the medulla oblongata and pons, mainly of the

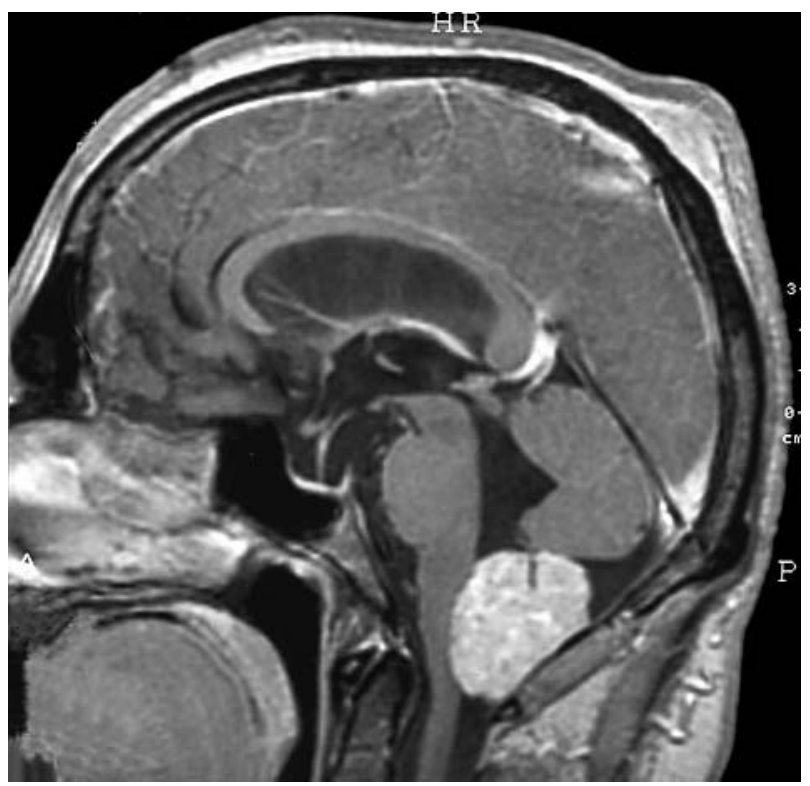

Figure 1 Preoperative magnetic resonance, post-contrast sagittal $\mathrm{Tl}$ weighted image: round, non-homogeneously enhancing tumour arising from the posterior aspect of the medulla oblongata occupies the region of the cisterna magna.

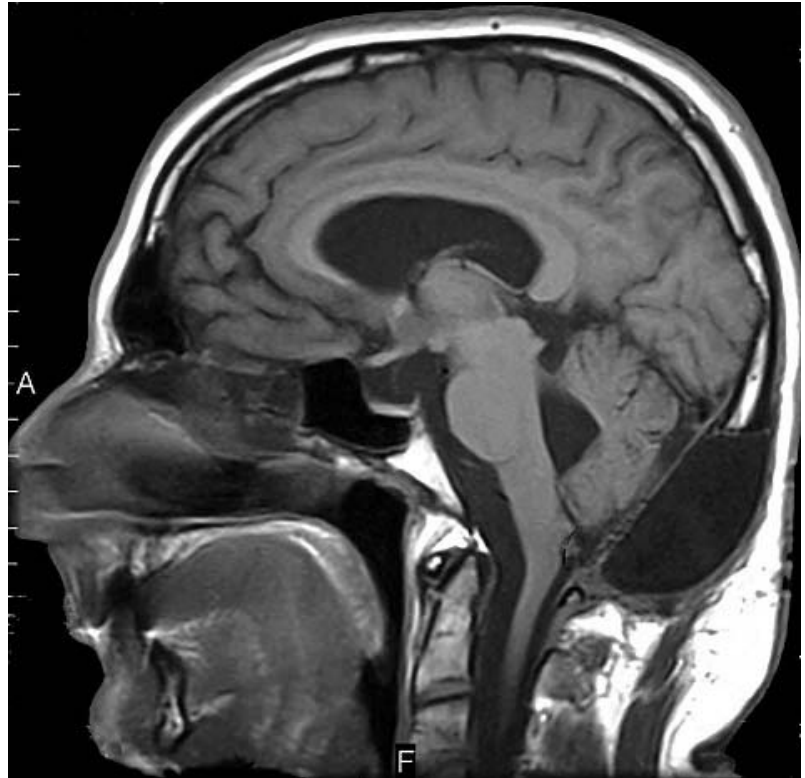

Figure 2 Postoperative magnetic resonance, postcontrast sagittal $\mathrm{T} 1$ weighted image: absence of residual tumour.

reticular formation and the ambiguous and solitary nuclei, resulting in damage to both the respiratory neurone group and the afferent pathways. Our case is the first reported where positional and sleep dyspnoea was caused by an exophytic brainstem tumour. The surgically documented infiltration of the posterior aspect of the medulla oblongata and the large tumour size probably caused episodic stretching of the underlying nervous structures by the tumour mass when the patient was lying down, resulting in transient impairment of the function of the respiratory centres and pathways. This is also confirmed by the occurrence of severe intraoperative oxygen desaturation caused by even a minimal upward mobilization of the tumour.

F Maiuri and M Esposito Dipartimento di Scienze Neurologiche, Clinica Neurochirurgica, Universitý Federico II, Via S. Pansini 5, Naples, Italy

Correspondence to: Professor F Maiuri; frmaiuri@unina.it Competing interests: none declared

\section{References}

1 Adelman S, Dinner DS, Goren H, et al. Obstructive sleep apnea in association with posterior fossa neurologic disease. Arch Neurol 1984;41:509-13.

2 Sheridan F, Scharf D, Henderson VW, et al. Lipomas of the mesencephalic tectum and rostral pons associated with sleep apnea syndrome. Clin Neuropathol 1990;9:152-56.

3 Valente S, De Rosa M, Culla G, et al. An uncommon case of brainstem tumor with selective involvement of the respiratory centers. Chest 1993; 103:1909-10.

4 Osanai S, lida Y, Nomura T. A case of unilateral brain-stem tumor and impaired ventilatory response. Nihon Kyobu Shikkan Gakkai Zasshi 1994;32:990-5.

5 Ito K, Murofushi T, Mizuno M, et al. Pediatric brain stem gliomas with the predominant symptom of sleep apnea. Int J Pediatr Otorhinolaryngol 1996;37:53-64.

6 Manning HL, Leiter JC. Respiratory control and respiratory sensation in a patient with a ganglioglioma within the dorsocaudal brainstem. Am J Respir Crit Care Med 2000;161:2100-6. 